\title{
Virtualized High Throughput Satellite Gateway with a Global Bandwidth Management Method
}

\author{
Haike Liu (D, Huajian Zhang, Kai Yang, and Jiali Li \\ Beijing Institute of Satellite Information Engineering, Beijing, China \\ Correspondence should be addressed to Haike Liu; ko5330@163.com
}

Received 25 November 2021; Revised 17 December 2021; Accepted 23 December 2021; Published 11 January 2022

Academic Editor: Hasan Ali Khattak

Copyright (c) 2022 Haike Liu et al. This is an open access article distributed under the Creative Commons Attribution License, which permits unrestricted use, distribution, and reproduction in any medium, provided the original work is properly cited.

\begin{abstract}
With the development of new satellite payload technology, in order to improve the utilization of system resources, research is based on software-defined network (SDN) and network function virtualization (NFV) gateway architecture. Based on this architecture, the system realizes global resource management and overall data distribution, which can solve the problem of resource allocation and maximum/minimum rate guarantee between different VNO terminals under different beams, different gateways, and different satellites. For this, a global bandwidth management method can be used which is mainly a process of management to control the traffic on a communication link. The proposed global resource management and control method can be based on the rate guarantee value of the $\mathrm{VNO}$ /terminal configured in the system as the basic limiting condition and reallocate the rate guarantee value limiting parameter according to the resource application status of the online terminal. The method can maximize the resource utilization of the entire satellite communication system and satisfy the resource request of the user terminal as much as possible.
\end{abstract}

\section{Introduction}

With the increasing demand for network communication and the development of satellite communication technology, the future communication network will be composed of satellite communication network and ground communication network. Satellite communication cannot be replaced in cross ocean and regional communication, international military communication, search and rescue, navigation and positioning, aviation communication, and other fields [1].

VHTS has the characteristics of multibeam and multigateway, as described in Figure 1. In order to support mobility, the system performs cross beam, cross gateway, and cross satellite handover technology [2]. Through the beam handover technology, the mobile terminal can realize wide area global roaming. Usually, satellite operators rent satellite bandwidth resources to virtual operators or charge according to the used bandwidth traffic. In order to ensure the service quality, satellite operators will set the total maximum rate limit and minimum guaranteed rate for each VNO; that is, the total rate of all terminals in VNO will not exceed the maximum rate, and the resources corresponding to the minimum guaranteed rate can be obtained permanently. However, due to the characteristics of VHTS, VNO terminals may be distributed in different geographical regions, corresponding to different satellite beams and gateways. Therefore, for distributed multigateway and multibeam systems, how to ensure the QoS guarantee requirements of terminal group consistency will be a key technology.

Based on the virtualization technology of satellite gateway, this paper constructs a new cloud gateway architecture, realizes the overall resource allocation function of the whole network, and solves the problem of global bandwidth management of satellite communication system.

VHTS system consists of gateway, satellite, and terminal. As the central part of the satellite communication system, the gateway undertakes the access and control of the terminal and needs to complete the signalling and data transmission between the ground and the satellite. Therefore, the resource allocation of the gateway plays a particularly important role in the efficient utilization of the satellite 


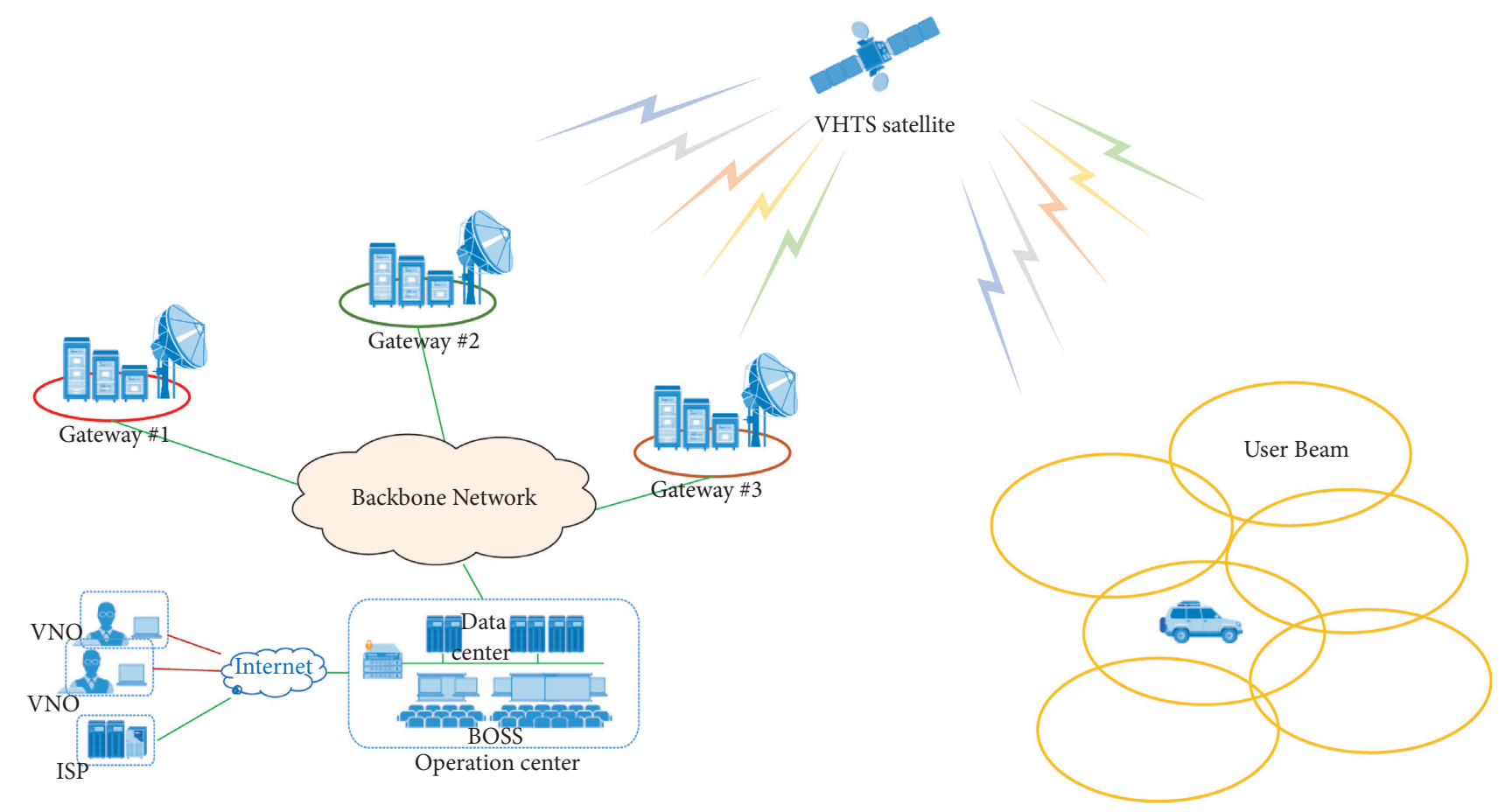

FIGURE 1: VHTS communication system.

system [3]. If the virtualization technology can be fully applied to the gateway, the comprehensive resource scheduling ability of the satellite system can be greatly improved. NFV/SDN and other key technologies can be used to play a prominent role in satellite-ground integration, focusing on solving the problem of satellite-ground segmentation of network functions [4]. At the same time, through the centralized and virtualized management and design of gateway, the reasonable allocation and efficient management of channel resources can be realized, and flexible configuration and on-demand allocation can be carried out. Solve the problems of integration and backward compatibility between different communication networks. It solves many problems due to the uneven geographical distribution of users, the tidal effect of users' all day network access time distribution, and the unequal resource usage of different gateways. The rest of the paper is arranged as follows: Section 2 contains the virtualized gateway and resource management methodology while Section 3 focuses on the GBM bandwidth allocation methodology. Similarly, Section 4 throws light on simulation results and finally Section 5 is the concluding section.

\section{Virtualized Gateway and Resource Management Methodology}

2.1. Virtualized Gateway Architecture. In order to solve the problem of global bandwidth management of multibeam and multigateway VHTS system, firstly, a new gateway architecture is designed based on software define network (SDN) and network function virtualization (NFV), which separates the network function hardware entity from the control, that is, separation of control plane and forwarding plane of equipment. Break the limitations of traditional integrated network architecture in network configuration, network services, cross system interconnection, and so on. It is conducive to the flexible networking and collaborative application of satellite systems and realizes the seamless switching of data with the ground network to realize flexible information transmission and distribution.

Network functions and network infrastructure can be abstracted and virtualized. Combined with the effective mapping mechanism from virtual resources to physical resources, the differences between physical networks and devices are shielded. The upper application no longer needs to deal directly with the lower physical devices, so as to realize the transparent management of the lower network. Relying on the abstraction, isolation, and migration functions of NFV, the gateway based on SDN/NFV can customize the required network services according to the personalized needs of users, so as to realize the software adaptability and flexible adaptation for new services in the future.

All functional entities of the satellite gateway are realized through software. The whole baseband unit is virtualized and connected with the remote RF unit through the forward transmission link. Each of these functions can be realized through independent virtual devices to form a satellite virtual gateway. In such an environment with all virtualization functions, the satellite network functions of concretization, operation, control, and management can be realized through intelligent processing of cloud server to meet the task requirements of users at the application layer, as shown in Figure 2. This realizes the satellite cloud wireless 


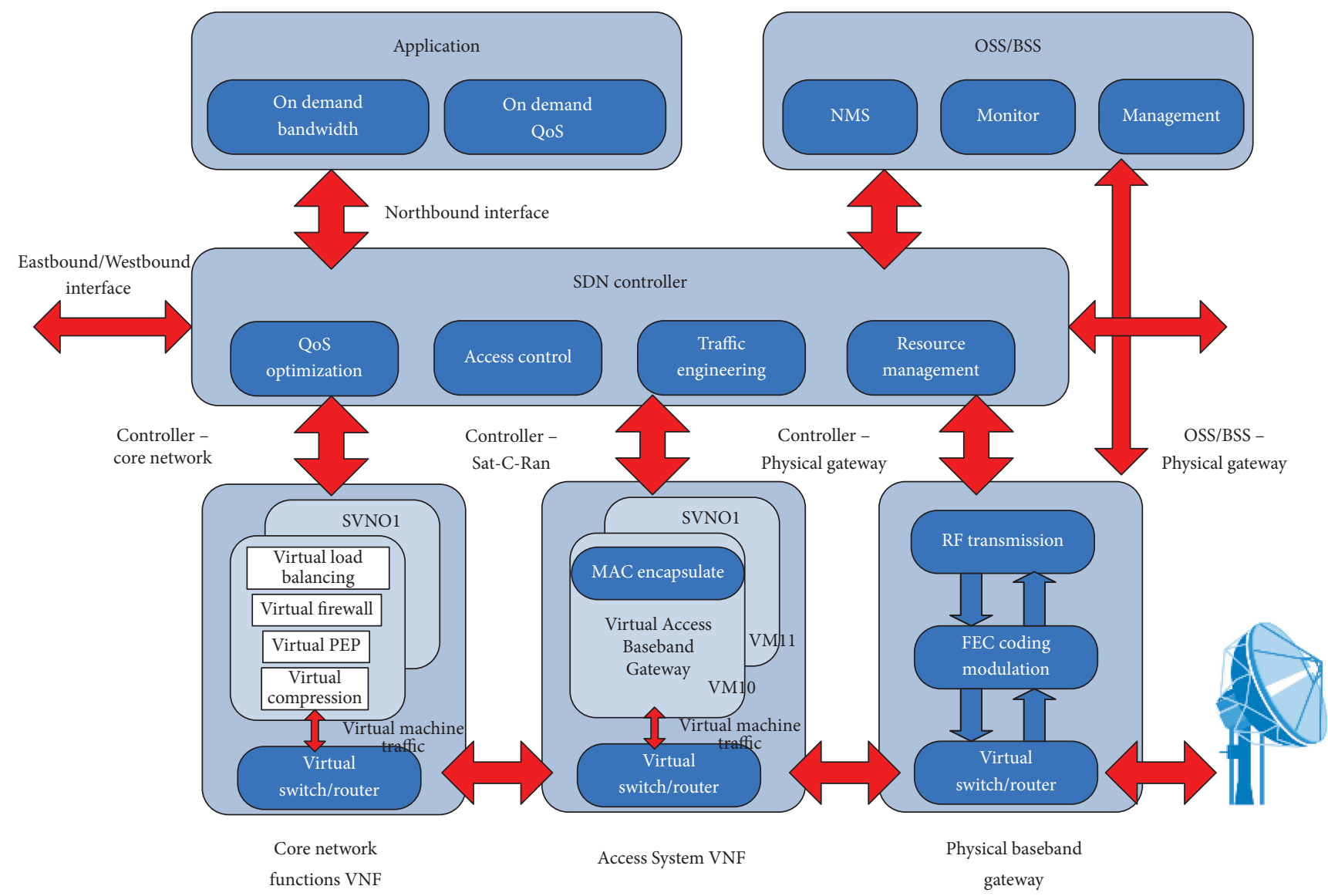

FIgURE 2: Sat-C-RAN architecture based on SDN/NFV [5].

access network (Sat-C-RAN) architecture. The application of cloud environment hides the complexity of underlying technology and network layer. Virtual network operators can fully automate the delivery and allocation of user services at the control layer, realize rapid and flexible allocation of network resources, or combine network virtual applications on demand to support elastic and flexible service transmission.

Sat-C-RAN of satellite network is based on Cloud Architecture and SDN to realize efficient resource utilization, flexible resource sharing. and low cost. Through these, it can adapt to different communication systems and does not rely on physical special equipment. These virtualized satellite access networks and terrestrial broadband access networks based on C-RAN architecture realize the integration of architecture and function as shown in Figure 3. The ground network system and different satellite systems realize resource integration at the control level through SDN and NFV. The unified management and control layer can carry out global resource allocation and overall data distribution and transmission for various access networks [6].

Under this architecture, the satellite network and ground network can be integrated at different layers, as shown in Figure 3. The connection at the lowest physical network facility level can be realized through ground physical network facilities and satellite physical network facilities (gateways, terminals, etc.) through ground network connection. The management control layer of the ground network can be directly integrated with the management control layer of the satellite network or connected to a common SDN controller to run a common and consistent communication protocol and management mechanism. At the service level, different networks can receive user service requests and task requirements through their user oriented northbound interface. The service integration control is connected with the management control layer, which can realize the overall planning of service tasks on the integrated network management control [7].

2.2. Global Bandwidth Management Method. Based on the above Sat-C-RAN architecture, a unified global bandwidth management function is constructed at the system control level to solve the QoS consistency guarantee of VNO groups under VHTS with multibeams and multigateways.

The VHTS is designed with multispot beams and multigateways, which emerged in commercial network communications for global coverage. However, because of the high number of small spot beams, a VHTS uses geographically spread gateways and deploys to cover more geographic regions. In a wholesaler-reseller network, VNO buys satellite bandwidth from the HNO at beam-level capacity [8]. Each beam is managed by a gateway to provide the subscribed resource to each VNO. Due to the localized 


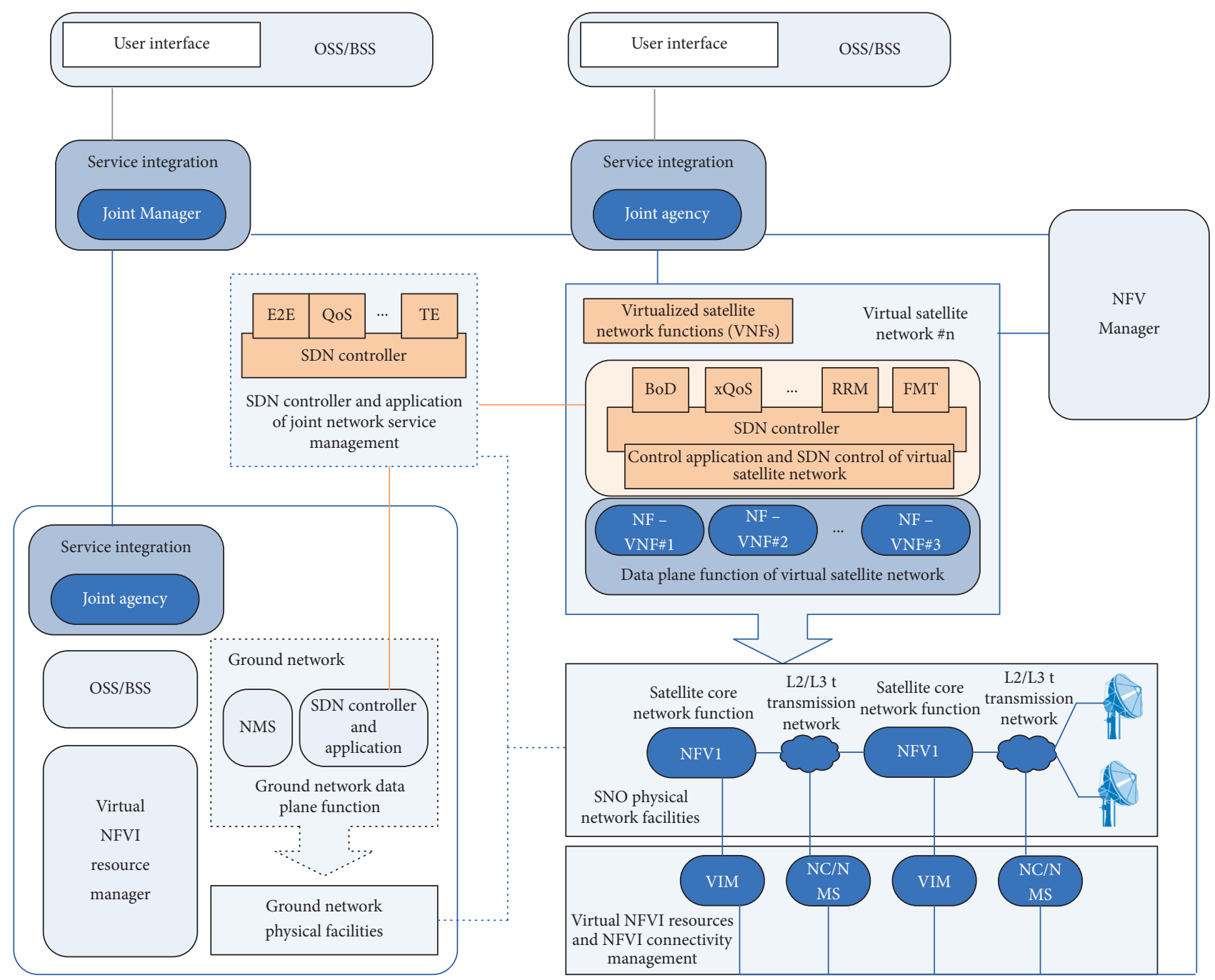

FIgURE 3: Architecture model of the satellite communication integrated network based on software definition.

bandwidth management for specific beams, the traditional bandwidth distribution method is no longer applicable to distributed gateway. Based on the Sat-C-RAN, we propose a GBM to perform a proportional fair distribution to one or more VNOs on the specific beam. The GBM will be run in a centralized control plane for all Sat-C-RANs and a VNO may subscribe to the system level aggregated bandwidth across multiple satellites or at an individual satellite level.

Figure 4 depicts a VNO subscription, which may include the multiple beams, gateways, and satellites. Each gateway serves to specific beams by performing bandwidth partition within one or more VNOs. The bandwidth partition is divided among the VNOs with respect to a GBM configuration. An Inroute Bandwidth Manager (IBM) and Outroute Bandwidth Manager (OBM) will be implemented in the control plane of each Sat-C-RAN. Firstly, an IBM receives the inroute capacity request from active terminals which subscribe to actual VNO on specific beams and periodically forwards to the GBM. The inroute demand may include an aggregate or current status information per beam per VNO. Then the GBM computes an inroute demand from IBM, which is distributed to the minimum and maximum bandwidth guaranteed to each VNO and sends back to the IBM for capacity allocating based on a VNO subscription retrieved from a data store and inroute demand.

\section{GBM Bandwidth Allocation Methodology}

3.1. Bandwidth Distribution Algorithm. The present section discloses the system and method for bandwidth management for the HNO to distribute the satellite capacity to one or more VNOs. Herein, each terminal is associated with a VNO, and each VNO has a bandwidth limitation where a VNO is subscribed to a system level aggregated bandwidth for a global subscription across multiple spot beams and multiple satellites for inroute (uplink) direction. Moreover, each VNO is served for one or more beams, and the beams also served from different gateways, as described in Figure 5. A gateway may include an IBM to provide beam bandwidth sharing for inroute connectivity.

At beam level, an IBM may gather and forward inroute demand from all active terminals to the GBM including an aggregate and current capacity information per VNO on a 


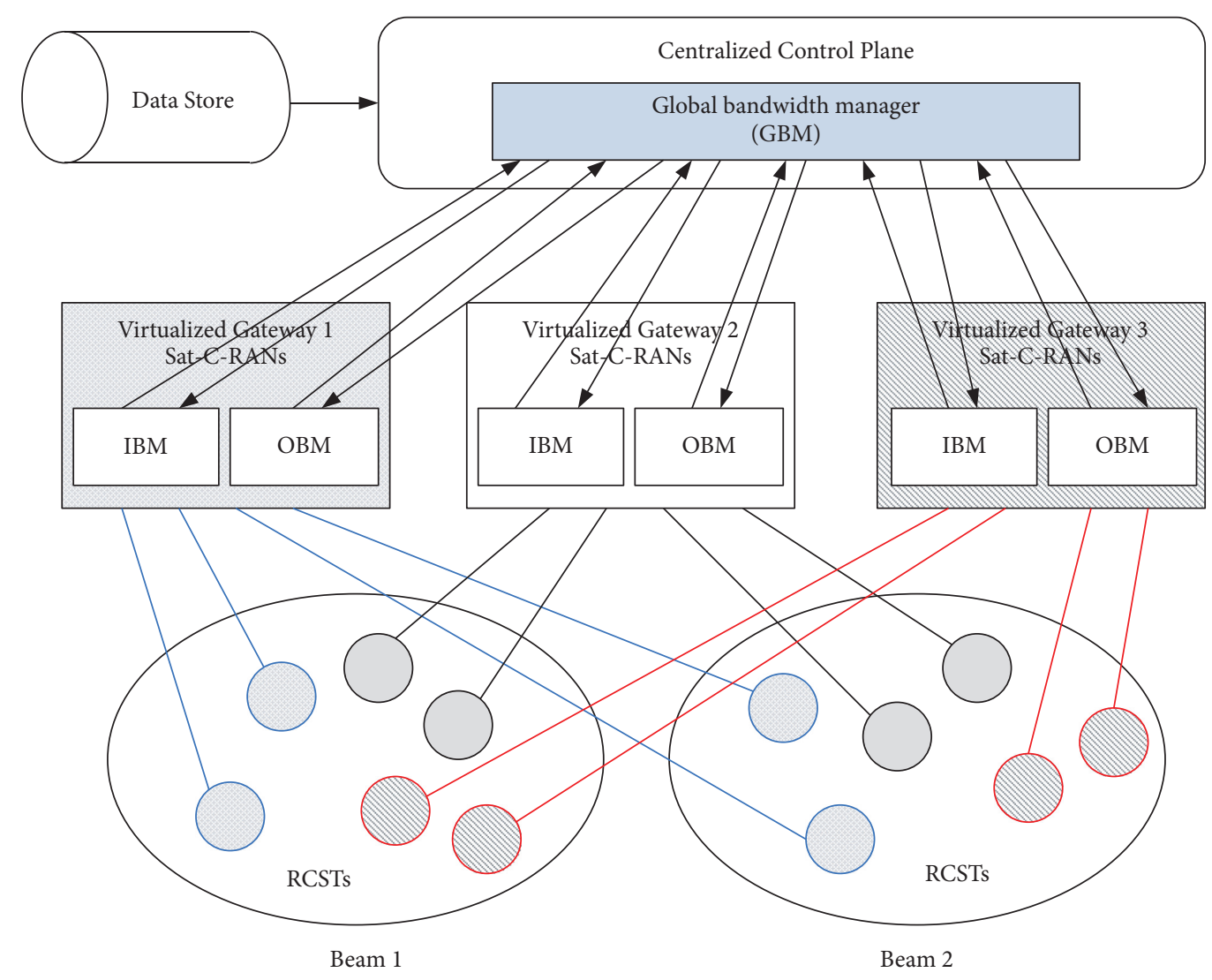

FIgURE 4: Global bandwidth management.

specific beam, where an IBM periodically sends bandwidth status report every superframe period [9]. Then, the GBM receives the inroute demands from all IBM and computes the bandwidth partition to each VNO. Finally, the GBM performed a proportional fair distribution based on multidimensional factors to calculate how much bandwidth is to be allocated to actual VNOs based on demands in different beams. It independently configured the CIR (committed information rate) and MIR (maximum information rate) bandwidth limited subscription methods to individual VNO and again to a terminal, respectively. We apply this condition to all $\mathrm{VNO}_{i}$ where $i$ th is the number of VNOs. Then, we give the condition as follows:

$$
\begin{aligned}
\mathrm{CIR}_{i} & \leq \mathrm{VNO}_{i} \leq \mathrm{MIR}_{i}, \\
\min _{i j}\left(T_{r}\right) & \leq T_{r} \leq \max _{i j}\left(T_{r}\right),
\end{aligned}
$$

where $\mathrm{CIR}_{i}$ is the minimum bandwidth guaranteed to a $\mathrm{VNO}_{i}$; $\mathrm{MIR}_{i}$ is the maximum bandwidth assigned to $\mathrm{VNO}_{i} ; \min _{i j}\left(T_{r}\right)$ is the minimum bandwidth guaranteed to a terminal in a $\mathrm{VNO}_{i}$ on a beam $j$; $\max _{i j}\left(T_{r}\right)$ is the maximum bandwidth assigned to a terminal of a $\mathrm{VNO}_{i}$ on a beam $j$. The CIR is a way to guaranteed minimum bandwidth to a VNO. Typically, it is low enough so that the system can share a bandwidth pool with many other terminals, at least a part of this specification rate, no matter how busy the link gets. The MIR is the theoretical maximum bandwidth, which may allocate when the terminals ask for a rate higher than CIR and lower than or equal to MIR as bandwidth becomes available. In case the terminals ask for a rate higher than MIR, then system will consider it as the request equal to MIR. Thus, the CIR and MIR configuration for bandwidth management methods ensures that the requested capacity from all terminals does not exceed the network capacity.

3.1.1. Minimum Bandwidth Distribution. A VNO subscribes to a system level minimum bandwidth, which is guaranteed to a VNO and normally available in network for all VNOs. The GBM receives the aggregated guaranteed bandwidth from active terminals in a VNO which is forwarded by the IBM and then runs a proportional fair distribution to a particular VNO demand. Let $\mathrm{MIN}_{i j}$ be the aggregate guaranteed to an $\mathrm{VNO}_{i}$ on a beam $j$ at any instant of time, and let $\mathrm{CIR}_{i}$ be the system level minimum bandwidth of a $\mathrm{VNO}_{i}$; then the minimum subscription distribution of that VNO may be calculated as

$$
\operatorname{MIN}_{i j}=\frac{\mathrm{CIR}_{i} * \min _{i j}}{\sum_{j=1}^{J} \min _{i j} \text { over all beams } j \text { to } J},
$$

where $\min _{i j}$ is the summation of the minimum bandwidth guaranteed to all terminals in a $\mathrm{VNO}_{i}$ on a beam $j$, and the summation of $\min _{i j}$ over all beams $j$ to $J$ does not exceed the VNO's global system level subscription. In (2), the aggregated minimum bandwidth $\mathrm{MIN}_{i j}$ allocated the VNOs 


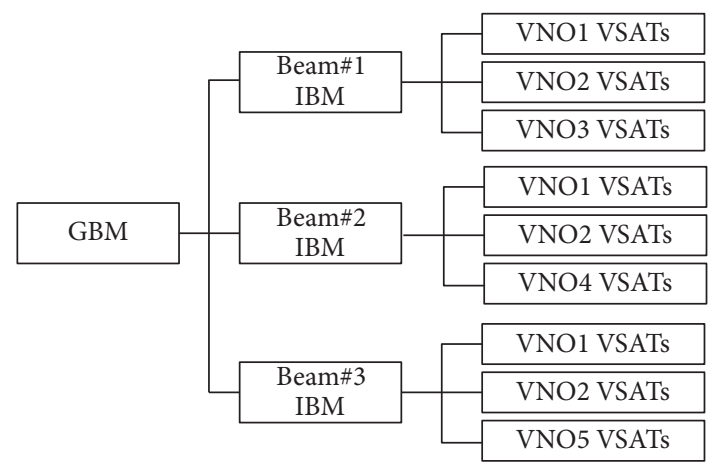

Figure 5: Communication flow epoch of an inroute chain.

always available to its subscriber terminals and it depended on how much bandwidth was guaranteed to a terminal. In the satellite network, each RCST can get at least a set amount of the minimum bandwidth guaranteed to an entity even in the waste case of the link connection.

3.1.2. Maximum Bandwidth Distribution. A VNO subscribes to a system level for maximum bandwidth and may treat it as the best effort bandwidth. Every flow control epoch, the GBM receives the information of aggregated maximum bandwidth subscription from active terminals in $\mathrm{VNO}_{i}$ on a beam $j$ and also the current traffic of inroute demands, separately. Let $\mathrm{MAX}_{i j}$ be the aggregated maximum subscription of all active terminals in a $\mathrm{VNO}_{i}$ on a beam $j$ at any instant of time and let $D_{i j}$ be the aggregated current demand from all active terminals of a $\mathrm{VNO}_{i}$ on a beam $j$. Let $\mathrm{MIR}_{i}$ be the system level maximum subscription of a $\mathrm{VNO}_{i}$, and then the maximum subscription distribution of that VNO is calculated as

$$
\operatorname{MAX}_{i j}=\frac{\operatorname{MIR}_{i} *\left(\alpha * \max _{i j}+(1-\alpha) * D_{i j}\right)}{\sum_{j=1}^{J}\left(\alpha * \max _{i j}+(1-\alpha) * D_{i j}\right) \text { over all beams } j \text { to } J} .
$$

Let $\max _{i j}$ be the summation of the maximum bandwidth assigned to all terminals in a $\mathrm{VNO}_{i}$ on a beam $j$; let $D_{i j}$ be the summation from all active terminals demands in a $\mathrm{VNO}_{i}$ on a beam $j$. A static factor $\alpha$ may bias between the configuration for maximum subscription and actual demand in the above calculation. By default, the value of $\alpha$ may give more weight to traffic demand and may be set as $0.1,0.2,0.3$, or the like. The present equation (3) may enforce that the summation of the maximum bandwidth distribution $\operatorname{MAX}_{i j}$ over all beams $j$ to $J$ of a VNO does not exceed global system level for maximum subscription based on the configuration constraints. Moreover, this equation is based on every terminals demand $D_{i j}$ and if the available capacity is greater than the requested capacity, then the system will allocate bandwidth as much as the terminals demand and also provide more weight.

3.1.3. Beam-Level Bandwidth Distribution. A beam $j$ has its own bandwidth $\mathrm{BW}_{j}$ to share with all subscribers VNOs on a specific coverage area. It may include the IBM to manage and share this bandwidth to each VNO demand, after calculating the minimum and maximum bandwidth aggregated to that VNO as the preresource. Then, at beam level, an IBM distributes $\mathrm{BW}_{j}$ to all VNOs. Let $P_{i j}$ be the prebandwidth to be allocated to a $\mathrm{VNO}_{i}$ on a beam $j$ and it is calculated as follows:

$$
P_{i j}=\frac{\mathrm{BW}_{j} * \mathrm{VNO}_{i j}}{\sum \mathrm{VNO}_{i j} \text { all VNOs on a beam } j} .
$$

Herein, let $\mathrm{VNO}_{i j}=\mathrm{MIN}_{i j}+\mathrm{MAX}_{i j}$ be the summation of the minimum and maximum bandwidth aggregated to a $\mathrm{VNO}_{i}$ on a beam $j$. Due to the demand assignment to all VNOs, then the total bandwidth requests can be higher than beam level. Using (4), the summation of the resources allocated to each VNO $P_{i j}$ must be less than or equal to $\mathrm{BW}_{j}$ at beam level.

Therefore, we propose a dynamic bandwidth allocation based on each spot beam demand that was considered only when the total traffic demand exceeds the system capacity. In the real network, each spot beam will request different traffic demand. The proposed methods claim that the algorithm for bandwidth management in the satellite network applies to the HNO on how to distribute the total capacity to multiple VNOs at a system level limitation with respect to a global subscription, where each VNO receives the allocation bandwidth based on demands. Moreover, the allocation bandwidth to all VNOs will not exceed the system capacity.

3.2. Fair Bandwidth Allocation Algorithm. The VNO has capability to manage its own partition of network capacity and allocate independently to its subscriber terminals (RCSTs) [10]. We propose a fair resource allocation algorithm to allocate a bandwidth requested to each RCST, individually. Herein, a fair distribution of $P_{i j}$ resources (timeslots) among $R$ entities is achieved by the following maximization problem, where $r \in R$ is the number of terminals (RCST) and $N_{r}$ is the number of timeslots assigned to $T_{r}, \forall r \in R$ :

$$
\begin{aligned}
& \max _{N_{1}, \ldots, N_{R}} \prod_{r=1}^{R} N_{r} \quad \text { subject to } \prod_{r=1}^{R} N_{r} \\
& \quad \leq P_{i j} \min _{i j}\left(N_{r}\right) \leq N_{r} \leq \max _{i j}\left(N_{r}\right),
\end{aligned}
$$

where $\min _{i j}\left(N_{r}\right)$ is the minimum number of timeslots guaranteed to a terminal $T_{r}$ and $\max _{i j}\left(N_{r}\right)$ is the maximum number of timeslots assigned to terminal $T_{r}$. This algorithm is invoked at the gateway to generate the TBTP2 for the return link based on the MF-TDMA scheme, which is divided into frequency and time division for burst transmission. The burst rate size is defined by designed payload which considers modulations, code rate, burst length, and other characteristics; see Table 1 [11]. For bandwidth allocation, we proposed a fixed MFTDMA channel structure, where each timeslot can transmit a burst rate. Then, calculate as below with three conditions. 


$$
N_{r}=\left\{\begin{array}{l}
\left\lceil\frac{C R_{i j}\left(T_{r}\right)}{R_{b}}\right\rceil \text { if } \min _{i j}\left(N_{r}\right) \leq N_{r} \leq \max _{i j}\left(N_{r}\right), \\
\min _{i j}\left(N_{r}\right) \text { if } N_{r} \leq \min _{i j}\left(N_{r}\right), \\
\max _{i j}\left(N_{r}\right) \text { if } N_{r} \geq \max _{i j}\left(N_{r}\right) .
\end{array}\right.
$$

The $C R_{i j}\left(T_{r}\right)$ is the requested capacity of a terminal $T_{r}$ (Mbps) and $R_{b}$ is the burst rate per timeslot (byte). Herein, each terminal requests bandwidth based on its demand in random selection. But, the timeslots assigned to each terminal will stand between the minimum and maximum number of timeslots conditions, where the quantities of timeslots allocation to $r$ entity must be greater than or equal to the minimum number of timeslots guaranteed to each entity; otherwise, it must be less than or equal to maximum number of timeslots and requested capacity.

As illustrated in Figure 6, it is possible to achieve the solution in a graphic way, by simply filling a container accordingly with guaranteed resources and demands with an amount $P_{i j}$ of water. Since (5) is solvable, the solution firstly assigns the minimum amounts (namely, "pale water") and then "fairly" distributes the rest (namely, "strong water"). In this case, the solution is generally computed for a real-valued problem, but it is possible to obtain the particularization to the integer case, simply assuming one extra resource (round up) to a subgroup of users, sharing the same number of resources, and round down the remaining ones [12].

In case the requested timeslots quantities from active terminals are smaller than or equal to the available timeslots, then the NCC allocates the timeslots to each terminal as much as the requested timeslots quantity and allocates free timeslots to each terminal in proportion to estimate the increased number of packets arriving at a transmission queue of each terminal. This is expressed as $\sum_{r=1}^{R} N_{r} \leq P_{i j}$. On the contrary, when the requested timeslot quantities of all active terminals are larger than the available timeslots number $P_{i j}$ and the NCC compares the requested timeslot quantity in a $\mathrm{VNO}_{i j}$ on a beam $j$ as $\sum_{r=1}^{R} N_{r} \geq P_{i j}$, then it cannot allocate the timeslots demand to each entity. In this case, we need to reduce all terminals demand in fairness. Let $N_{T S}(\lambda)$ be the number of timeslots demands from all active terminals after the reducing percentage $\lambda$ and can be calculated as the equation given as follows:

$$
N_{T S}(\lambda)=\sum_{r=1}^{R}\left(N_{r} \cdot \lambda\right) \leq P_{i j},
$$

where $\lambda=P / \sum_{r=1}^{R} N_{r}$ with $0.5 \leq \lambda \leq 0.9$, which means that at least each terminal can get $50 \%$ of its request. In the fair resource distribution, all entities need to reduce their demand in the same $\lambda$. After reducing all terminals demands, then the NCC normally can assign the requested timeslots to each terminal. Moreover, the network capacity can be reallocated and reassigned in a period of update time for every superframe. The requested timeslots can assign and allocate to each entity when the available resources in MFTDMA channel structure have enough space to fit with.
After an entity allocated, then the next entity will assign continuously. Then, the available resources can be defined as follows:

$$
T_{r}=N_{r} \leq P_{i j}-\sum_{r=1}^{R} N_{r-1} ; N_{0}=0 .
$$

Equation (8) is used to find the available timeslots for the next terminals location in the MF-TDMA, which start first in time-frequency and continue to allocate until the last timefrequency block. Then, the terminal location in TBPT2 can be expressed as the following equation:

$$
\begin{aligned}
T_{r} & =(1,2, \ldots, m) \operatorname{or}(T S 1, T S 2, \ldots, T S m), \\
m & =k+\sum_{r=1}^{R} N_{r-1} ; k=1,2, \ldots, N_{r} .
\end{aligned}
$$

Equation (9) is used to find the optimal timeslots location in MF-TDMA structure and can allocate the requested timeslots of the terminals continuously, and then the assigned timeslots to a terminal must not overlap in time and frequency block. This equation will allocate the requested timeslot with first-come first-serve. Each timeslot allocated to the terminal can be defined as $\left(f_{m}, t_{n}\right)$, $f_{m} \in F C s ; t \in T_{t r f}$, to know the exact location of carrier frequency and time for data transmission.

This method provides more efficiency for timeslot allocation in fairness, which assigns the requested timeslots to each terminal demand. The timeslots are allocated continuously without overlapping in time and frequency block, with a fasted, reliable, and stable system capacity assignment.

\section{Simulation Results}

4.1. Simulation Implementation. For the system configuration, we apply for the geostationary bent pipe satellite communication, where three GEO satellites can provide virtual worldwide coverage. Each beam coverage has its own bandwidth with a gateway to manage this bandwidth and share with the amount of terminals on its coverage served by multiple VNOs. For the global satellite communication system, we mention three beams' coverage consisting of 10,000 terminals and served by 5 different VNOs. Each VNO has 2000 terminals independently (see Table 2).

Each VNO can serve to one or multiple beams; for example, the VNO1 and VNO2 serve to all beams with different amount of entities; but, VNO3, VNO4, and VNO5 individually served only a beam, respectively. As mentioned in previous section, the GBM configured the minimum and maximum bandwidth limit condition at the system level subscription, wherein each VNO has the same CIR and MIR bandwidth configuration to determine how much bandwidth to be allocated over the CIR and up to the MIR values. We configure $2 \mathrm{Gbps}$ for minimum bandwidth guaranteed to a VNO (CIR) and $4 \mathrm{Gbps}$ for maximum bandwidth assigned to a VON (MIR), where each terminal receives at least $1 \mathrm{Mbps}$ and up to 3 Mbps. 
TABLE 1: The reference waveforms for linear modulation format class bursts.

\begin{tabular}{|c|c|c|c|c|c|}
\hline Waveform ID & Burst length & Payload length (bytes) & Payload length (symbols) & Mapping scheme & Code rate \\
\hline 1 & 664 & 38 & 456 & QPSK & $1 / 3$ \\
\hline 6 & 536 & 96 & 512 & QPSK & $3 / 4$ \\
\hline 8 & 536 & 115 & 460 & 8PSK & $2 / 3$ \\
\hline 12 & 536 & 194 & 466 & 16QAM & $5 / 6$ \\
\hline 21 & 1616 & 599 & 1438 & 16QAM & $3 / 4$ \\
\hline 31 & 1868 & 59 & 1416 & BPSK & $1 / 3$ \\
\hline
\end{tabular}

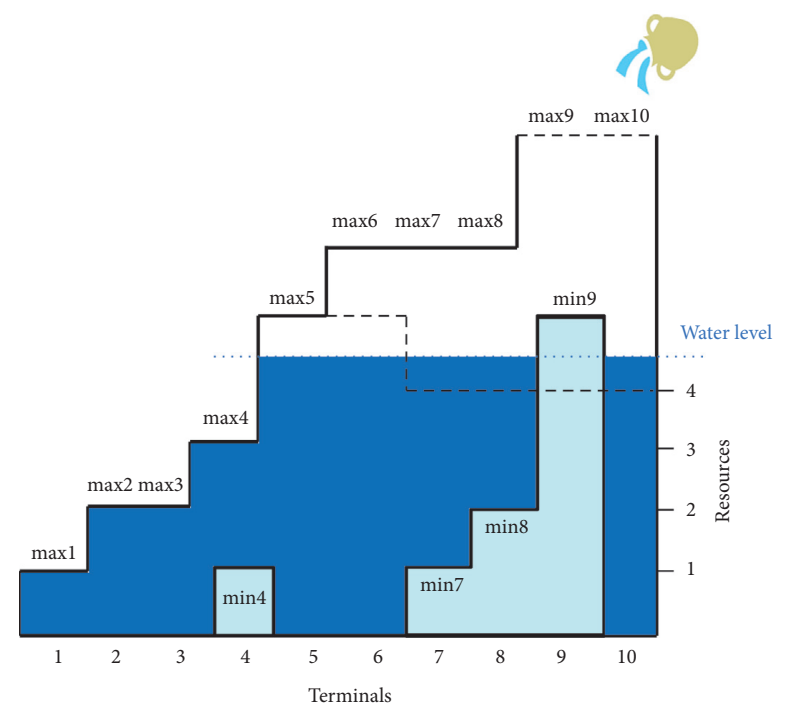

FIGURE 6: Fair resource distribution solution.

TABLE 2: Parameters configuration for the system simulation.

\begin{tabular}{|c|c|c|c|c|}
\hline \multicolumn{5}{|c|}{$\begin{array}{c}\text { GBM CIR_ } i=2 \text { Gbps } \leq \text { VNO_is } i \leq \mathrm{MIR} \_i=4 \text { Gbps } \\
\min \_i j=1 \mathrm{Mbps} \leq T \_r \leq \max \_i j=3 \mathrm{Mbps}\end{array}$} \\
\hline \multirow{6}{*}{ Beam $j$} & Beam \#1 & VNO1 & VNO2 & VNO3 \\
\hline & Terminals & $1000 \mathrm{~T}$ & $1000 \mathrm{~T}$ & $2000 \mathrm{~T}$ \\
\hline & Beam \#2 & VNO1 & VNO2 & VNO4 \\
\hline & Terminals & $500 \mathrm{~T}$ & $500 \mathrm{~T}$ & $2000 \mathrm{~T}$ \\
\hline & Beam \#3 & VNO1 & VNO2 & VNO5 \\
\hline & Terminals & $500 \mathrm{~T}$ & $500 \mathrm{~T}$ & $2000 \mathrm{~T}$ \\
\hline
\end{tabular}

4.2. Bandwidth Distribution Results. Using the parameters configuration in Table 2, we simulate to solve the previous equations for bandwidth distribution. For the maximum bandwidth distribution $\mathrm{MAX}_{i j}$, it depends on all active terminals demand $D_{i j}$. Herein, each terminal requests a capacity in random selection from 0.5 to $4 \mathrm{Mbps}$. In the above calculation, $\alpha$ is a static factor that provides bias between configured maximum and actual demand to provide more weight to a terminal demand, for example, by setting $\alpha$ to 0.3 . Then, we get the results as in Table 3 .

Table 3 is the result of bandwidth distribution to each VNO at system level limited for global bandwidth subscription. Actually, the VNO demand is higher than the total network capacity; this is because each terminal requested a capacity based on its demand. But, the allocation bandwidth consumption $P_{i j}$ assigned to all VNOs does not exceed the network capacity. Herein, $P_{i j}$ is the prebandwidth that must allocate to its subscriber terminals. Depending on the CIR and MIR configuration to a particular VNO, the minimum and maximum bandwidth distributed to actual VNO have not exceeded the global bandwidth subscription at a system level limited. These results show that the proposed method is very efficient for bandwidth distribution and to maximize utilization of the satellite network capacity.

4.3. Fair Timeslot Allocation Results. There are three steps in MF-TDMA resources allocation algorithm. The first step is to determine the requested bandwidth from active terminals; the second step is to determine the quantities of timeslots that fit with actual terminal demand; the third step is to locate the assignment timeslots into the entire bandwidth with the specific carrier frequencies and times block. In DVB-RCS2 standard, an RCST sends a message for capacity request to NCC, after it has scheduled how to request timeslots. In particular, when an RCST has data to transmit, it first explicitly requests the capacity to NCC using DAMA technique. However, the bandwidth request from active terminals may change over time and different terminals may request different amount of traffic types. Herein, we give a restriction to every terminal to request once a capacity in random selection from ranges $0.5 \mathrm{Mbps}$ to $4 \mathrm{Mbps}$, as illustrated in Figure 7.

As illustrated in Figure 7, it depicts the trade-off between capacity requested (CR) and capacity assignment (CA) to all active terminals in a VNO with 50 terminals. Each terminal 
TABLE 3: Bandwidth distribution results.

\begin{tabular}{|c|c|c|c|c|c|c|}
\hline \multicolumn{7}{|c|}{ Bandwidth distribution } \\
\hline Beam $j$ & $\mathrm{BW}_{j}(\mathrm{Gbps})$ & $\mathrm{VNO}_{i}$ & Terminals & $\mathrm{MIN}_{i j}(\mathrm{Mbps})$ & $\operatorname{MAX}_{i j}(\mathrm{Mbps})$ & $\mathrm{P}_{i j}(\mathrm{Mbps})$ \\
\hline \multirow{3}{*}{ Beam 1} & \multirow{3}{*}{10} & $\mathrm{VNO}_{1}$ & 1000 & 1000 & 2010.17 & 2515.90 \\
\hline & & $\mathrm{VNO}_{2}$ & 1000 & 1000 & 1954.39 & 2469.28 \\
\hline & & $\mathrm{VNO}_{3}$ & 2000 & 2000 & 4000 & 5014.80 \\
\hline \multirow{3}{*}{ Beam 2} & \multirow{3}{*}{10} & $\mathrm{VNO}_{1}$ & 500 & 500 & 1010.595 & 1672.86 \\
\hline & & $\mathrm{VNO}_{2}$ & 500 & 500 & 1019.383 & 1682.59 \\
\hline & & $\mathrm{VNO}_{4}$ & 2000 & 2000 & 4000 & 6644.536 \\
\hline \multirow{3}{*}{ Beam 3} & \multirow{3}{*}{10} & $\mathrm{VNO}_{1}$ & 500 & 500 & 979.23 & 1642.59 \\
\hline & & $\mathrm{VNO}_{2}$ & 500 & 500 & 1026.22 & 1694.77 \\
\hline & & $\mathrm{VNO}_{5}$ & 2000 & 2000 & 4000 & 6662.62 \\
\hline
\end{tabular}

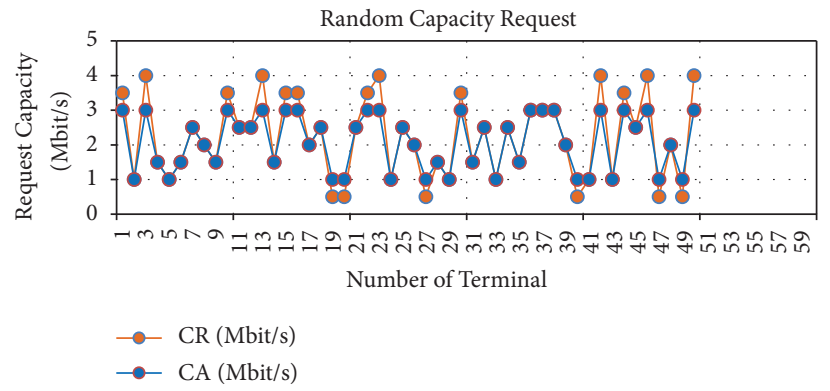

FIGURE 7: Capacity request and capacity assignment in random selection.

makes the capacity request in random selection with a range from 0.5 to $4 \mathrm{Mbps}$. But, in reality a terminal can request more capacity based on its demand. This simulation shows the results of (6) with some restrictions. Even though a terminal requested a capacity less than $1 \mathrm{Mbps}$ - for example, it requested $0.5 \mathrm{Mbps}$ - then the capacity assigned to that terminal is $1 \mathrm{Mbps}$; when a terminal requested a capacity greater than $3 \mathrm{Mbps}$-for example, it requested $4 \mathrm{Mbps}$ - the capacity assigned to a terminal is just $3 \mathrm{Mbps}$. Both cases depended on the CIR and MIR bandwidth limited configuration guaranteed to a terminal. Therefore, the requested bandwidth assigned to all terminals does not exceed the available network capacity.

We treat the timeslots as the main resources that need to be allocated to each actual terminal based on its capacity requirement. After a terminal has calculated the amount of timeslots, it then sends a message to the NCC, where the NCC is in charge of bandwidth allocation for the return channel timeslot transmission and informs all RCSTs of allowable slots by generating the TBTP2. Each RCST reads TBTP2 and transmits data during the allocated timeslots. Herein, TBTP2 indicates at which time or burst is allowed to send data and per timeslot can transmit one burst rate. Each burst rate can transmit 599 bytes for 16QAM with the code rate $3 / 4$; see Table 1 .

Timeslots allocation unit determines the quantities of timeslots according to the terminal demand. As shown in Figure 8, for example, when an RCST requested $1 \mathrm{Mbps}$ of capacity, then it needed 209 timeslots; when it requested $2.5 \mathrm{Mbps}$, then it needed 522 timeslots for burst transmission defined by frequency, bandwidth, starting time, and duration.

As mentioned above, the bandwidth requested from active terminals is allocated entirely into a fixed MF-TDMA from one superframe to another superframe, which is still the inputs problem. To solve this issue, we propose a fair resource allocation algorithm invoked at the NCC to assign the requested capacity by generating TBTP2, wherein this algorithm examines a fixed MFTDMA to search for a contiguous set of unused timeslots large enough to accommodate the requested bandwidth. If sufficient resources are found, then they are allocated into MF-TDMA structure with a multiplicity of frequencies and time block that was organized in superframes, frames, and timeslots. Here, we design a fixed MF-TDMA with multiple frequency carriers $f_{m}$, each of which is used for $2 \mathrm{Mbps}$ that contain 32 superframes and each superframe has 48 timeslots. Thus, the gateway uses the total timeslots $f_{m} \bullet 32 \bullet 48$ to locate all terminals demand in TBTP2.We only show the allocation of 10 terminals that randomly request the capacity and assign continuously in a fixed MF-TDMA, as seen in Figure 9.

Figure 9 depicts the location of terminals into a fixed MF-TDMA structure based on its current demand. For example, RCST1 requested 627 timeslots which are located from timeslot number 1 in the first frequency and time till slot number 627; the next RCST2 requested 209 and then was allocated continuously from slot number 628 till slot 836; and the next RCST will be allocated continuously with none overlapping in frequency and 


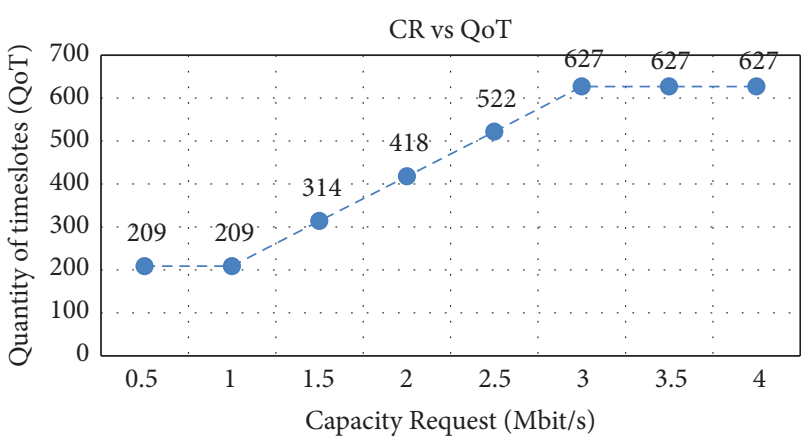

Figure 8: Capacity requested (Mbps) in the number of timeslots.

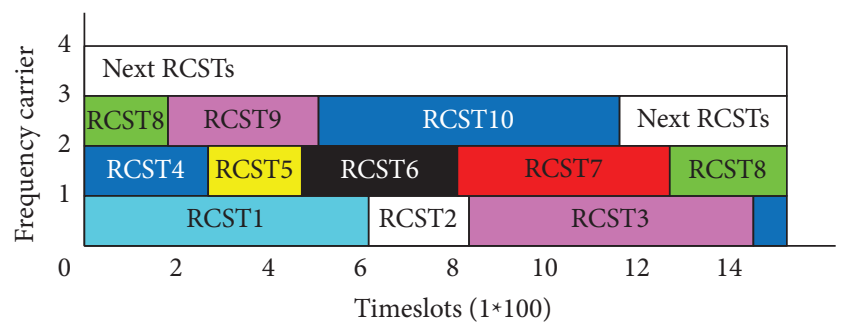

FIgURE 9: Timeslots allocation in a fixed MF-TDMA.

time. As a result, this means first-come and first-serve that allocates in a short time, is fast and reliable, and reduces the data transfer delay.

\section{Conclusion}

For satellite communication system, the traditional network architecture cannot meet the requirements no matter from the perspective of economic or technological progress. Therefore, a virtualization-based Sat-C-RAN architecture is proposed. The C-RAN network architecture effectively realizes the sharing of baseband resources between gateway through the centralized processing and allocation of baseband resources, so as to improve the utilization of baseband resources. The number of baseband resources required is reduced, and the system construction cost and energy loss during operation are effectively reduced. The resources of satellite communication system are limited. Fully applying virtualization technology to gateway stations can greatly improve the comprehensive scheduling and utilization of satellite communication system resources.

Based on the Sat-C-RAN architecture, the presented algorithm of bandwidth distribution for the HNO has the ability to calculate how much bandwidth to be allocated to one or more VNOs across multiple beams, gateways, and satellites with respect to a global bandwidth subscription. This restriction is to ensure that the bandwidth consumption assigned to a particular VNO will not exceed the network capacity, even though their traffic demand is higher than the available resources. In a satellite network, our method is very efficient for resource management to maximize the utilization of network capacity, especially providing fairness for bandwidth distribution, fast resource allocation, and reliable and short time delay for data transfer. However, due to the localized bandwidth management of each beam, some of a VNO's demands can be subject to congestion on a beam, while there is spare capacity on another beam that the $\mathrm{VNO}$ also subscribes to. As such, the localized bandwidth management cannot respond dynamically to the need of $\mathrm{VNO}$ overall. This means that a satellite network can share its own capacity among gateways belonging to the same network and under control of the same NCC, but cannot share the network capacity for intersatellite communication systems [13]. For that reason, this research can present an innovation implementation of the resource allocation mechanism based on VNOs demand, which support dynamic bandwidth allocation for intersatellites communication networks and also for resource assignment that can allocate into a dynamic MF-TDMA. More importantly, dynamic bandwidth resource sharing between intersatellites network involves many system- and technology-dependent aspects.

5.1. Future Work. This work can be further elaborated at a major scale; in particular, some sort of method or algorithm can be implemented to lower the congestion while dealing with localized bandwidth management.

\section{Data Availability}

The data used to support the findings of this study are included within the article.

\section{Conflicts of Interest}

The authors declare that they have no conflicts of interest.

\section{References}

[1] G. Araniti, I. Bisio, M. De Sanctis, A. Orsino, and J. Cosmas, "Multimedia content delivery for emerging 5g-satellite networks," IEEE Transactions on Broadcasting, vol. 62, no. 1, pp. 10-23, 2016.

[2] ViaSat ViaSat-3: https://www.viasat.com/products/high-capacitysatellites.

[3] U. Park, H. W. Kim, D. S. Oh, and B. J. Ku, "A dynamic bandwidth allocation scheme for a multi-spot-beam satellite system,” ETRI Journal, vol. 34, no. 4, pp. 613-616, 2012.

[4] F. Riffel and R. Gould, "Satellite ground station virtualization: secure sharing of ground stations using software defined networking," in Proceedings of the 2016 Annual IEEE Systems Conference (SysCon), IEEE, Orlando, FL, USA, April 2016.

[5] T. Ahmed, E. Dubois, R. F. Jb Dupé, P. Gélard, and N. Kuhn, "Software-defined satellite cloud ran," International Journal of Satellite Communications and Networking, vol. 36, no. 1, 2018.

[6] G. Li, H. Zhou, B. Feng, G. Li, and X. Qi, "Horizontal-based orchestration for multi-domain sfc in sdn/nfv-enabled satellite/terrestrial networks," China Communication, vol. 15, no. 5, pp. 87-101, 2018.

[7] R. Ferrus, O. Sallent, T. Ahmed, and R. Fedrizzi, "Towards SDN/NFV-enabled satellite ground segment systems: end-toEnd Traffic Engineering use case," in Proceedings of the IEEE International Conference on Communications Workshops, IEEE, Paris, France, May 2017.

[8] L. Kuang, X. Chen, C. Jiang, H. Zhang, and S. Wu, "Radio resource management in future terrestrial-satellite 
communication networks," IEEE Wireless Communications, vol. 24, pp. 81-87, 2017.

[9] M. K. Park and D. G. Oh, "DVB-RCS2 emulation system: to verify system level functions for multiple terminals," in Proceedings of the International Conference on Information \& Communication Technology Convergence, pp. 62-64, Jeju Island, South Korea, May 2015.

[10] J. M. R. Bejarano, C. M. Nieto, and F. J. Ruiz Pinar, "Mf-tdma scheduling algorithm for multi-spot beam satellite systems based on co-channel interference evaluation," IEEE Access, vol. 7, pp. 4391-4399, 2018.

[11] Etsi En 301 545-2, "Digital video broadcasting (DVB); second generation DVB interactive satellite system (DVB-RCS2); Part 2: lower layers for satellite standard," DVB Document A155-2, February-2019.

[12] X. Zhu, C. Jiang, L. Kuang, Z. Zhao, and S. Guo, “Two-layer game based resource allocation in cloud based integrated terrestrial-satellite networks," IEEE Transactions on Cognitive Communications and Networking, vol. 6, no. 2, pp. 509-522, 2020.

[13] A. Kyrgiazos, B. Evans, P. Thompson, and N. Jeannin, "Gateway diversity scheme for a future broadband satellite system," in Proceedings of the Advanced Satellite Multimedia Systems Conference (ASMS) and 12th Signal Processing for Space Communications Workshop (SPSC), pp. 363-370, Vigo, Spain, September 2012. 